\title{
Generation of density perturbations at the end of inflation
}

\author{
Michael P. Salem* \\ California Institute of Technology, Pasadena, California 91125, USA
}

(Received 16 November 2005; published 22 December 2005)

\begin{abstract}
Recently a mechanism was proposed whereby the primordial density perturbations are generated at the end of inflation. We continue the analysis of the proposed model of this mechanism and calculate the maximum extent to which the density perturbations produced via this model can dominate over those of the standard inflationary paradigm. In addition, we provide a straightforward variation of this model which allows for greater amplification of the density perturbations. Finally, we show that a variation in the implementation of the original model results in significant non-Gaussianities in the resulting spectrum of density perturbations. The level of non-Gaussianities can be made to saturate the current observational bound.
\end{abstract}

DOI: 10.1103/PhysRevD.72.123516

PACS numbers: $98.80 . \mathrm{Cq}$

\section{INTRODUCTION}

Measurements of the cosmic microwave background radiation [1] have revealed a highly uniform background with relatively scale-free superhorizon perturbations on the order of a few parts in $10^{5}$. A possible source of these perturbations is found in the quantum fluctuations of one or more light scalar fields during an early epoch of inflation [2,3]. This is because in the (quasi-) de Sitter space of inflationary expansion, a quantum fluctuation in a scalar field evolves according to classical equations of motion after its wavelength exceeds the rapidly decreasing Hubble length [4]. A fluctuating scalar field may then be converted into an energy density perturbation via a variety of proposed mechanisms.

For example in the standard inflationary paradigm [3], inflation is driven by the potential energy of a single slowly-rolling scalar field. In this case the inflaton can be viewed as a unique clock parameterizing the evolution of the early universe. Therefore fluctuations in the inflaton translate into fluctuations in the duration of inflation. Since the energy density of the Universe redshifts more rapidly after inflation than during inflation, this results in energy density fluctuations on surfaces of constant scale factor after inflation.

More recently, it was proposed that energy density perturbations could result from fluctuations in light scalar fields that do not contribute significantly toward the inflationary dynamics. For example, in the "curvaton" scenario [5] (see also [6]) a light scalar field dubbed the curvaton receives fluctuations. After inflation, the curvaton evolves as a massive fluid and therefore redshifts more slowly than the radiative products of reheating. If the curvaton eventually dominates the energy density of the Universe and then decays, density perturbations result because the duration of curvaton domination depends on the fluctuating curvaton. The inhomogeneous reheating scenario [7] also

*Electronic address: salem@theory.caltech.edu achieves density perturbations by varying the duration that a massive fluid dominates the energy density of the Universe. In this case the duration of domination is modulated via a decay width that depends on some fluctuating light scalar field. Other ways to modulate the duration of massive fluid's domination have been proposed in [8]. These ideas recently have been reviewed in [9].

Another major variant on these proposals was offered recently in [10] (note however that a similar idea was previously used in [11]). Whereas in the standard picture the duration of inflation is influenced by the fluctuations in the inflaton as relevant modes leave the horizon, in this picture the duration of inflation is influenced by fluctuations in the inflaton when inflation ends. This can occur, for example, if inflation ends at an inflaton value that depends upon some other field that receives fluctuations during inflation. In [10] a specific model was used to demonstrate that this scenario can lead to significant amplification of the density perturbations that result from slow-roll inflation.

Here we continue the analysis of that model and calculate the maximum extent to which the resulting density perturbations can dominate over those of the standard inflationary paradigm. We also explore the sensitivity of this result to the tuning of model parameters. In addition, we provide a straightforward variation upon this model which allows for greater amplification of the density perturbations. Finally, we show that a slight variation in the implementation of the original model allows for significant non-Gaussianities in the spectrum of density perturbations. The level of non-Gaussianities can be made to saturate the current observational bound.

This paper is organized as follows: In Sec. II we summarize the mechanism of [10] and establish a convenient formalism and notation. Section III describes the specific model introduced in [10], and in Sec. IV we analyze this model in greater detail. Section V introduces two variations upon this model and studies their consequences. Discussion and concluding remarks are provided in Sec. VI. 


\section{BACKGROUND}

We parameterize metric perturbations using the curvature perturbation on surfaces of constant scale factor, otherwise known as the Bardeen variable $\zeta$ [12]. This corresponds to writing the interval

$$
d s^{2}=-d t^{2}+a^{2}(t) e^{2 \zeta(t, \mathbf{x})} \gamma_{i j}(t, \mathbf{x}) d x^{i} d x^{j},
$$

where $a$ and $\zeta$ are chosen to give $\gamma_{i j}$ unit determinant. In this work we ignore tensor perturbations, which allows us to write $\gamma_{i j}(t, \mathbf{x})=\delta_{i j}$ since scalar field fluctuations carry no anisotropic stress (for a review of cosmological perturbation theory see [13]).

The proposal of [10] is most easily studied within the socalled $\delta N$ formalism of [14] (see [15] for recent extensions). In this formalism it is noted that on superhorizon scales the change in $\zeta$ between two surfaces of constant time is given by the fluctuation in the number of e-folds of universal expansion between those surfaces. We choose the initial time $t_{0}$ to be some time when $\zeta=0$ so that we can write [14]

$$
\zeta(t, \mathbf{x})=\delta N(t, \mathbf{x})
$$

where

$$
N(t, \mathbf{x}) \equiv \ln \left[\frac{a(t) e^{\zeta(t, \mathbf{x})}}{a\left(t_{0}\right)}\right]
$$

is the number of e-folds of expansion between $t$ and $t_{0}$.

We limit our attention to the case where inflation is driven by a single inflaton. Then fluctuations in $N$ come only from the value of the inflaton when its wavelength becomes larger than the Hubble length, denoted $\phi_{k}$, and the value of the inflaton at the end of inflation, denoted $\phi_{\mathrm{e}}$. Thus the number of e-folds of universal expansion is $N\left(\phi_{k}, \phi_{\mathrm{e}}\right)$, and

$$
\delta N \simeq \frac{\partial N}{\partial \phi_{k}} \delta \phi_{k}+\frac{\partial N}{\partial \phi_{\mathrm{e}}} \delta \phi_{\mathrm{e}} .
$$

That the power spectrum for $\zeta$ is observed to be predominantly Gaussian allows us to neglect higher order terms that might appear in the expansion of Eq. (4).

In the standard picture, inflation ends when the slow-roll conditions are violated. For single field inflation this happens at a unique value $\phi_{\mathrm{e}}$ and therefore the second term in Eq. (4) is zero. For inflation to end at varying values of $\phi_{\mathrm{e}}$ requires the addition of at least one other field. For example, with an additional field $\sigma$ it is possible that $\phi_{\mathrm{e}}(\sigma)$ depends upon $\sigma$ and inflation ends within a range of $\phi_{\mathrm{e}}$ given by

$$
\delta \phi_{\mathrm{e}} \simeq \frac{\partial \phi_{\mathrm{e}}}{\partial \sigma} \delta \sigma .
$$

If the masses of the scalar fields are much less than the Hubble rate the fields acquire a constant power spectrum of fluctuations $\mathcal{P}_{\delta \phi_{k}}=\mathcal{P}_{\delta \sigma_{k}}=\left(H_{k} / 2 \pi\right)^{2}$, where $H_{k}$ is the Hubble rate at the time the mode $k$ exits the horizon [16]. Thus the power spectrum for $\zeta$ is

$$
\mathcal{P}_{\zeta} \simeq \frac{H_{k}^{2}}{4 \pi^{2}}\left[\left(\frac{\partial N}{\partial \phi_{k}}\right)^{2}+\left(\frac{\partial N}{\partial \phi_{\mathrm{e}}} \frac{\partial \phi_{\mathrm{e}}}{\partial \sigma_{k}}\right)^{2}\right] .
$$

The density perturbations generated at the end of inflation will dominate over those produced from the standard picture when

$$
\frac{\partial N}{\partial \phi_{\mathrm{e}}} \frac{\partial \phi_{\mathrm{e}}}{\partial \sigma}>\frac{\partial N}{\partial \phi_{k}} .
$$

Note that $\partial N / \partial \phi_{k} \neq \partial N / \partial \phi_{\mathrm{e}}$ since the former probes the dependence of $N$ on $\phi$ deep in the inflationary epoch while the latter probes the dependence of $N$ on $\phi$ near the end of inflation.

\section{THE SPECIFIC MODEL}

The above ideas were demonstrated in [10] using a specific model described by the potential

$$
\begin{aligned}
V(\phi, \chi, \sigma) & =\frac{1}{4 g}\left(m_{\chi}^{2}-g \chi^{2}\right)^{2}+\frac{1}{2} m_{\phi}^{2} \phi^{2}+\frac{1}{2} \lambda_{\phi} \phi^{2} \chi^{2} \\
& +\frac{1}{2} \lambda_{\sigma} \sigma^{2} \chi^{2}+V_{\sigma}(\sigma) .
\end{aligned}
$$

As was noted in [10], this is the original hybrid inflation model [17] but with interactions involving an additional light scalar field $\sigma$ added with the last two terms.

Hybrid inflation assumes the initial conditions of chaotic inflation $[17,18]$. However, during the early stages of hybrid inflation the field $\chi$ is pushed to zero much faster than $\phi$ (and likewise faster than $\sigma$ in the above model). Then $\phi$ becomes a slowly-rolling inflaton with inflation assisted by the vacuum energy $m_{\chi}^{4} / 4 g$. As in [10] we assume that the self interaction of $\sigma$ represented by the term $V_{\sigma}$ does not contribute significantly toward the inflationary dynamics.

The field $\chi$ is pinned to the origin until the curvature in its potential, $\partial^{2} V / \partial \chi^{2}$, becomes negative. In [10] a scenario is described in which inflation ends abruptly as $\chi$ rolls away from the origin. This happens when

$$
\frac{\partial^{2} V}{\partial \chi^{2}}=\lambda_{\phi} \phi_{\mathrm{e}}^{2}+\lambda_{\sigma} \sigma_{\mathrm{e}}^{2}-m_{\chi}^{2}=0
$$

Therefore if $\sigma$ receives fluctuations $\delta \sigma$, inflation ends at field values $\phi_{\mathrm{e}}$ that vary according to

$$
\delta \phi_{\mathrm{e}} \simeq-\frac{\lambda_{\sigma} \sigma_{\mathrm{e}}}{\lambda_{\phi} \phi_{\mathrm{e}}} \delta \sigma .
$$

Referring back to Eq. (6), we see the density perturbations resulting from fluctuations $\delta \phi_{\mathrm{e}}$ dominate over those resulting from $\delta \phi_{k}$ when

$$
R \equiv \frac{\lambda_{\sigma}^{2} \sigma_{\mathrm{e}}^{2}}{\lambda_{\phi}^{2} \phi_{\mathrm{e}}^{2}}\left(\frac{\partial N / \partial \phi_{\mathrm{e}}}{\partial N / \partial \phi_{k}}\right)^{2}=\frac{\lambda_{\sigma}^{2} \sigma_{\mathrm{e}}^{2}}{\lambda_{\phi}^{2} \phi_{\mathrm{e}}^{2}} \frac{\epsilon_{k}}{\epsilon_{\mathrm{e}}}>1 .
$$

In the second equation we have used that the first slow-roll parameter can be written

$$
\epsilon \equiv \frac{m_{\mathrm{pl}}^{2}}{2}\left(\frac{1}{V} \frac{\partial V}{\partial \phi}\right)^{2}=\frac{1}{2 m_{\mathrm{pl}}^{2}}\left(\frac{\partial N}{\partial \phi}\right)^{-2} .
$$


An important consequence of this mechanism is that the spectral tilt is given by the tilt in the spectrum of fluctuations $\delta \sigma$, as opposed to $\delta \phi$. This gives a tilt which is independent of the second slow-roll parameter $\eta[10,16]$. Therefore $\eta$ is not directly constrained by observation. In addition, since $\dot{\epsilon} \simeq 2 H \epsilon(2 \epsilon-\eta)$ it appears as if $\epsilon$ may decrease significantly during the course of inflation if $\eta \gg$ $\epsilon$. Thus it was suggested in [10] that the condition of Eq. (11) is easily satisfied.

However, one might note that $R$ is proportional to $\epsilon_{k}$ which according to this mechanism is constrained by observational bounds on the spectral tilt. These give $\epsilon_{k} \lesssim$ 0.02 [1] in the absence of cancellations [10]. In addition, we do not expect $\epsilon_{\mathrm{e}}$ and $\phi_{\mathrm{e}}$ to be independent of each other. Thus it is worthwhile to investigate Eq. (11) in greater detail in order to determine precisely what limits the extent to which the density perturbations of this model can dominate over those produced via the standard inflationary paradigm.

\section{A MORE DETAILED ANALYSIS}

We now consider the model of Eq. (8) in greater detail in order to clarify the constraints on $R$ as given by Eq. (11). Consistent with the analysis of [10] we ignore the contribution of $V_{\sigma}$ toward the vacuum energy. As described above, the $\chi$ field rolls away from the origin when $\phi$ takes the value

$$
\phi_{\mathrm{e}}=\sqrt{\frac{m_{\chi}^{2}-\lambda_{\sigma} \sigma_{\mathrm{e}}^{2}}{\lambda_{\phi}}} \approx \frac{m_{\chi}}{\sqrt{\lambda_{\phi}}} .
$$

To explain the second approximation, first note that we require $\lambda_{\sigma} \sigma_{\mathrm{e}}^{2}<m_{\chi}^{2}$ in order to avoid $\chi$ remaining trapped at the origin after $\phi$ reaches zero. Meanwhile, the precise value of $\sigma_{\mathrm{e}}$ is a stochastic variable constrained by conditions independent of $\lambda_{\sigma}$ and $m_{\chi}$. Thus to ensure that the desired dynamics are typical of this model requires we set $m_{\chi}^{2} / \lambda_{\sigma} \gg \sigma_{\mathrm{e}}^{2}$ for typical values of $\sigma_{\mathrm{e}}$.

For inflation to last until $\phi=\phi_{\mathrm{e}}$ but end abruptly when $\chi$ rolls away from the origin requires that the term $m_{\chi}^{4} / 4 g$ dominate the energy density at the end of inflation and that $m_{\chi}^{2}$ be much greater than the Hubble rate at the end of inflation. ${ }^{1}$ These conditions, respectively, give the con-

\footnotetext{
${ }^{1}$ The other possibility is to set $\phi_{\mathrm{e}}=m_{\chi} / \sqrt{\lambda_{\phi}}$ to be greater than the value of $\phi$ for which inflation ends when the potential of $\phi$ dominates. Since in this case inflation ends when $\phi$ is of the order of the Planck mass [18], this requires a Planck scale $m_{\chi}$ unless $\lambda_{\phi} \gg 1$. Nevertheless, an analysis similar to that which follows gives

$$
R \simeq \frac{\phi_{k}^{2}}{\sigma_{\mathrm{e}}^{2}}\left(\frac{\lambda_{\sigma} \sigma_{\mathrm{e}}^{2}}{m_{\chi}^{2}}\right)^{2}
$$

where $\phi_{k}$ is $\phi$ evaluated 60 e-folds before the end of inflation. It will be seen that this is no better than the result obtained in the scenario analyzed in greater detail in what follows.
}

straints

$$
\frac{2 g}{\lambda_{\phi}} \frac{m_{\phi}^{2}}{m_{\chi}^{2}} \ll 1, \quad \frac{1}{12 g} \frac{m_{\chi}^{2}}{m_{\mathrm{pl}}^{2}} \ll 1,
$$

where $m_{\mathrm{pl}}$ denotes the reduced Planck mass. The first constraint of Eqs. (15) allows us to write

$$
\frac{\partial N}{\partial \phi_{\mathrm{e}}}=\frac{V}{m_{\mathrm{pl}}^{2}}\left(\frac{\partial V}{\partial \phi_{\mathrm{e}}}\right)^{-1} \simeq \frac{m_{\chi}^{4}}{4 g \phi_{\mathrm{e}} m_{\phi}^{2} m_{\mathrm{pl}}^{2}} \approx \frac{\sqrt{\lambda_{\phi}} m_{\chi}^{3}}{4 g m_{\phi}^{2} m_{\mathrm{pl}}^{2}} .
$$

Meanwhile

$$
\frac{\partial N}{\partial \phi_{k}}=\frac{1}{\phi_{k} m_{\phi}^{2} m_{\mathrm{pl}}^{2}}\left(\frac{m_{\chi}^{4}}{4 g}+\frac{1}{2} m_{\phi}^{2} \phi_{k}^{2}\right) .
$$

Plugging these values into Eq. (11) gives

$$
R \approx \frac{\lambda_{\sigma}}{4 g^{2}}\left(\frac{\lambda_{\sigma} \sigma_{\mathrm{e}}^{2}}{m_{\chi}^{2}}\right) m_{\chi}^{6} \phi_{k}^{2}\left(\frac{m_{\chi}^{4}}{2 g}+m_{\phi}^{2} \phi_{k}^{2}\right)^{-2} .
$$

The quantity $\phi_{k}$ is the value of the inflaton when the mode $k$ exits the particle horizon. For cosmological scales of current interest this happens about 60 e-folds of inflation prior to the end of inflation.

In order to find the maximum value for $R$, we find the value of $\phi_{k}$ that maximizes $R$ and set the parameters $g, m_{\chi}$, and $m_{\phi}$ such that the mode $k$ exits the horizon 60 e-folds prior to inflation. This is equivalent to finding the balance between the vacuum energy $m_{\chi}^{4} / 4 g$ and the potential energy $m_{\phi}^{2} \phi^{2}$ that maximizes $R$. The result is

$$
\phi_{k}=\frac{1}{\sqrt{2 g}} \frac{m_{\chi}^{2}}{m_{\phi}}
$$

with the constraint that the mode $k$ leaves the horizon at $N_{k} \approx 60$, with

$$
\begin{aligned}
N_{k} & =\frac{1}{4 m_{\phi}^{2} m_{\mathrm{pl}}^{2}}\left[\frac{m_{\chi}^{4}}{g} \ln \left(\frac{\sqrt{\lambda_{\phi}} \phi_{k}}{m_{\chi}}\right)+m_{\phi}^{2} \phi_{k}^{2}-\frac{m_{\phi}^{2} m_{\chi}^{2}}{\lambda_{\phi}}\right] \\
& \approx \frac{m_{\chi}^{4}}{8 g m_{\phi}^{2} m_{\mathrm{pl}}^{2}} \ln \left(\frac{\lambda_{\phi}}{2 g} \frac{m_{\chi}^{2}}{m_{\phi}^{2}}\right) .
\end{aligned}
$$

In the last expression we have used the constraints of Eqs. (15) to identify the most significant term. Putting this all together we find the maximum value of $R$ to be

$$
R \approx N_{k}\left(\frac{\lambda_{\sigma} \sigma_{\mathrm{e}}^{2}}{m_{\chi}^{2}}\right)^{2} \frac{m_{\mathrm{pl}}^{2}}{\sigma_{\mathrm{e}}^{2}}\left[\ln \left(\frac{\lambda_{\phi}}{2 g} \frac{m_{\chi}^{2}}{m_{\phi}^{2}}\right)\right]^{-1} .
$$

Before proceeding to study Eq. (21) we should check that producing the correct power spectrum normalization does not introduce any constraints that conflict with our present assumptions. Applying Eq. (6) to the scenario considered above we find that when density perturbations generated at the end of inflation dominate we have

$$
\mathcal{P}_{\zeta} \approx \frac{2}{\pi^{2}} N_{k} R\left(\frac{1}{12 g} \frac{m_{\chi}^{2}}{m_{\mathrm{pl}}^{2}}\right) \frac{m_{\chi}^{2}}{m_{\mathrm{pl}}^{2}}\left[\ln \left(\frac{\lambda_{\phi}}{2 g} \frac{m_{\chi}^{2}}{m_{\phi}^{2}}\right)\right]^{-1}
$$


To match observation we require $\mathcal{P}_{\zeta}$ to be very small, $\mathcal{P}_{\zeta} \approx\left(5 \times 10^{-5}\right)^{2}$ [1]. According to the second of Eqs. (15) the first term in parentheses is already constrained to be much less than order unity and in fact can be set as small as necessary to match observation. In addition we expect $m_{\chi}^{2} / m_{\mathrm{pl}}^{2}$ to be very small. Thus to set $\mathcal{P}_{\zeta}$ to match observation does not introduce any constraints in conflict with those of the above analysis.

We have written $R$ in the form of Eq. (21) in order to emphasize the maximum extent to which the perturbations produced at the end of inflation may dominate over those produced as cosmological scales exit the horizon. The first term is weakly constrained by the energy scale of inflation and is here taken to be $N_{k} \approx 60$. The first term in parentheses is constrained to be significantly less than unity as explained in the discussion below Eq. (13). Finally, the argument of the logarithm must be much greater than unity in order to satisfy the first constraint of Eqs. (15). Therefore the last term is less than unity. Thus for appropriately tuned parameters we might expect the product of these three factors to be a couple of orders of magnitude below unity.

However, Eq. (21) also contains a factor $m_{\mathrm{pl}}^{2} / \sigma_{\mathrm{e}}^{2}$. In (quasi-) de Sitter space a scalar field such as $\sigma$ evolves both according to its classical equation of motion and due to quantum fluctuations as modes leave the particle horizon. The net effect of this evolution is that the correlation function $\left\langle\sigma^{2}\right\rangle$ migrates toward a fixed value depending upon $V_{\sigma}$ and the Hubble rate $H$ [19]. For example, if inflation lasts long enough and if $V_{\sigma}=\frac{1}{2} m_{\sigma}^{2} \sigma^{2}$ then $\left\langle\sigma^{2}\right\rangle \sim H^{4} / m_{\sigma}^{2}$ [19]. Taking a typical value of $\sigma_{\mathrm{e}}$ to be $\sigma_{\mathrm{e}} \sim \sqrt{\left\langle\sigma^{2}\right\rangle}$ gives

$$
\frac{m_{\mathrm{pl}}^{2}}{\sigma_{\mathrm{e}}^{2}} \sim \frac{m_{\sigma}^{2}}{H^{2}} \frac{m_{\mathrm{pl}}^{2}}{H^{2}}
$$

The dynamics described above require $m_{\sigma}^{2} \ll m_{\phi}^{2} \ll H^{2}$, so the first term in Eq. (23) must be at least a few orders of magnitude below unity. However, the second term can be very large. Current observation gives $m_{\mathrm{pl}}^{2} / H^{2} \geqslant 10^{8}$ [20], which is more than sufficient to compensate for all the small factors in $R$ if parameters are tuned appropriately. Reducing the scale of inflation allows for greater values of $R$. Of course, our Hubble volume also could be a region of atypically small $\sigma_{\mathrm{e}}$. Finally, we may reduce $\sigma_{\mathrm{e}}$ to an arbitrarily small scale by considering $\sigma$ to be a pseudoNambu-Goldstone boson (see for example $[5,21]$ ). ${ }^{2}$ Thus

\footnotetext{
${ }^{2}$ An interesting scenario involves a pseudo-Nambu-Goldstone boson that ranges over a scale $\sim m_{\chi}$. Then $\lambda_{\sigma}$ may be a coupling of order unity and we obtain

$$
R \approx N_{k} \frac{m_{\mathrm{pl}}^{2}}{m_{\chi}^{2}},
$$

where we have dropped the logarithm and other factors of order unity. Clearly $R$ is much greater than unity in this case.
}

in a number of circumstances we expect the level of density perturbations generated at the end of inflation to be significantly larger than those produced when cosmological scales exit the horizon.

In Eq. (21) the parameters $g, m_{\phi}$, and $m_{\chi}$ are tuned such that scales of cosmological interest leave the horizon when $\phi_{k}$ is given by Eq. (19). To study the result of relaxing this tuning requires to invert Eq. (20) to obtain $\phi_{k}\left(N_{k}\right)$ and insert the result into Eq. (18) to obtain $R\left(N_{k}\right)$. This allows for the remaining parameters in $R$ to be freely varied while $R$ retains its original meaning; that is, that $R$ compares density perturbations produced at the end of inflation to those produced when cosmological scales exit the particle horizon.

Note that the first two terms in the brackets of Eq. (20) always dominate over the third term and that they are comparable to each other when $\phi_{k}$ is given by Eq. (19). Remember that $\phi_{k}$ is defined as the value of $\phi N_{k} \approx 60 \mathrm{e}-$ folds before the end of inflation. Decreasing $m_{\phi}$ slows the evolution of $\phi$ which therefore decreases $\phi_{k}$. In this case the first term in brackets becomes more important and inverting Eq. (20) gives

$$
\phi_{k}^{2} \approx \phi_{\mathrm{e}}^{2} \exp \left(8 N_{k} \frac{g m_{\phi}^{2} m_{\mathrm{pl}}^{2}}{m_{\chi}^{4}}\right) .
$$

For $\phi_{k}$ less than in Eq. (19) the important functional dependence of $R$ is $R\left(\phi_{k}\right) \propto \phi_{k}^{2}$. Thus we see that $R$ decreases exponentially when the ratio $g m_{\phi}^{2} m_{\mathrm{pl}}^{2} / m_{\chi}^{2}$ is decreased from its optimal value.

On the other hand, increasing $m_{\phi}$ quickens the evolution of $\phi$ and therefore increases $\phi_{k}$. In this case the second term in the brackets of Eq. (20) becomes more important. Inverting $N_{k}$ in this case gives $\phi_{k}^{2} \approx N_{k} m_{\mathrm{pl}}^{2}$ which is relatively independent of the model parameters. Therefore the magnitude of $R$ changes predominantly through its dependence upon $m_{\phi}^{2} \phi_{k}^{2}$ in the denominator of Eq. (18). Thus we see that significantly increasing the ratio $g m_{\phi}^{2} m_{\mathrm{pl}}^{2} / m_{\chi}^{4}$ from its optimal value results in a roughly proportional decrease in the size of $R$.

\section{GENERALIZING THE MODEL}

\section{A. Varying the potential for $\phi$}

According to Eq. (7), the generation of density perturbations at the end of inflation is most effective when the slow-roll parameter near the end of inflation is much less than when cosmological scales of interest exit the particle horizon. Thus to enhance the resulting perturbations we desire a potential for $\phi$ that decreases more steeply for $\phi$ deep in the inflationary epoch and decreases more gently for $\phi$ near the end of inflation. This can be accomplished by replacing the $\frac{1}{2} m_{\phi}^{2} \phi^{2}$ term in Eq. (8) with $\frac{1}{4} \lambda \phi^{4}$.

We analyze this scenario in exact analogy to the analysis in Sec. IV. Again we assume that the vacuum energy at the 
end of inflation is dominated by the $m_{\chi}^{4} / 4 g$ term. The calculations proceed just as in Sec. IV, and in the end we find

$$
R \simeq 108 N_{k}^{3}\left(\frac{\lambda_{\sigma} \sigma_{\mathrm{e}}^{2}}{m_{\chi}^{2}}\right)^{2}\left(\frac{m_{\mathrm{pl}}^{2}}{\sigma_{\mathrm{e}}^{2}}\right)\left(\frac{\lambda_{\phi} m_{\mathrm{pl}}^{2}}{m_{\chi}^{2}}\right)^{2}
$$

As described in Sec. IV, the term in the first set of parentheses is expected to be significantly less than unity. However the last term is expected to be greater than unity in order that $\chi$ becomes pinned to the origin in the early stages of inflation. In addition, the numerical prefactor $108 N_{k}^{3} \sim 10^{7}$ for $N_{k} \approx 60$. The discussion about the factor $m_{\mathrm{pl}}^{2} / \sigma_{\mathrm{e}}^{2}$ near the end of Sec. IV applies here. Thus we see the maximum level of density perturbations produced at the end of inflation can be greatly amplified by simply introducing a $\phi^{4}$ potential.

\section{B. Relaxing the constraint on $\boldsymbol{\lambda}_{\sigma}$}

In Sec. III we followed the assumption presented in [10] that near the end of inflation $\lambda_{\sigma} \sigma^{2}<m_{\chi}^{2}$ so that inflation ends before $\phi$ reaches zero. It is interesting to explore the consequences of lifting this assumption. If we do not demand that $\lambda_{\sigma} \sigma^{2}<m_{\chi}^{2}$, then for large enough $\lambda_{\sigma}$ or $\sigma$ the $\chi$ field is still pinned to the origin when $\phi$ reaches zero. We then expect inflation to continue with the $\sigma$ field rolling down its potential until the condition of Eq. (9) is met. At this point $\chi$ rolls away from the origin and abruptly initiates the end of inflation.

Nevertheless, if $m_{\phi} \ll H$ during inflation, then $\phi$ will retain a power spectrum of fluctuations given by $\mathcal{P}_{\delta \phi_{k}}=$ $\left(H_{k} / 2 \pi\right)^{2}$. Thus the fields $\sigma$ and $\phi$ have essentially changed places, with $\sigma$ playing the part of the inflaton and $\phi$ the fluctuating field. However, in this scenario $\phi$ has no homogeneous component and

$$
\delta \sigma_{\mathrm{e}}=-\frac{\lambda_{\phi}}{\lambda_{\sigma}} \frac{\delta \phi_{k}^{2}}{\sigma_{\mathrm{e}}}
$$

The density perturbations produced at the end of inflation are now entirely non-Gaussian.

It should be noted here that the original model described in Sec. III also results in non-Gaussianities. As described in [10], in that model the non-Gaussianities result from higher order terms in the expansion of $\delta N$ in Eq. (4). The situation we consider here is different in that the non-Gaussianities arise at leading order in the expansion of $\delta N$.

The level of non-Gaussianity can be parameterized with a term $f_{\mathrm{NL}}$ defined according to the equation

$$
\zeta=\zeta_{\mathrm{g}}-\frac{3}{5} f_{\mathrm{NL}} \zeta_{\mathrm{g}}^{2}
$$

where $\zeta_{\mathrm{g}}$ symbolizes a variable with a Gaussian spectrum [22]. In this case $\zeta_{\mathrm{g}}$ is the curvature perturbation produced via the standard inflationary paradigm,

$$
\zeta_{\mathrm{g}}=\frac{\partial N}{\partial \sigma_{k}} \delta \sigma_{k} .
$$

Here we have assumed for simplicity that scales of cosmological interest exit the horizon after $\phi$ has rolled to zero. Thus we treat the relevant stages of inflation as driven by the $\sigma$ field, assisted by the vacuum energy $m_{\chi}^{4} / 4 g$, with $\phi$ being a heavier field fluctuating about the origin. We neglect the non-Gaussian component of $\zeta$ produced when relevant scales exit the horizon as this is in general relatively small [23]. For comparison to observation it does not matter whether the non-Gaussian fluctuations are sourced by the same field as the Gaussian fluctuations [24]. Therefore we can combine Eq. (4), Eq. (28), and Eq. (29) to obtain

$$
\begin{aligned}
f_{\mathrm{NL}} & =-\frac{5}{3}\left(\frac{\partial N}{\partial \sigma_{\mathrm{e}}} \delta \sigma_{\mathrm{e}}\right)\left(\frac{\partial N}{\partial \sigma_{k}} \delta \sigma_{k}\right)^{-2} \\
& =\frac{5}{3} \frac{\lambda_{\phi}}{\lambda_{\sigma}} \frac{1}{\sigma_{\mathrm{e}}} \frac{\partial N}{\partial \sigma_{\mathrm{e}}}\left(\frac{\partial N}{\partial \sigma_{k}}\right)^{-2} .
\end{aligned}
$$

If we take $V_{\sigma}=\frac{1}{2} m_{\sigma}^{2} \sigma^{2}$, it is straightforward to calculate the largest possible $f_{\mathrm{NL}}$ by translating the arguments of Sec. IV. In particular, we note that $\sigma_{\mathrm{e}}=m_{\chi} / \sqrt{\lambda_{\sigma}}$ and that

$$
\frac{\partial N}{\partial \sigma_{\mathrm{e}}} \simeq \frac{1}{4 g} \frac{m_{\chi}^{4}}{\sigma_{\mathrm{e}} m_{\sigma}^{2} m_{\mathrm{pl}}^{2}}=\frac{\sqrt{\lambda_{\sigma}}}{4 g} \frac{m_{\chi}^{3}}{m_{\sigma}^{2} m_{\mathrm{pl}}^{2}} .
$$

Likewise, minimizing the factor $\left(\partial N / \partial \sigma_{k}\right)^{-2}$ gives $\sigma_{k}=$ $m_{\chi}^{2} / \sqrt{2 g} m_{\sigma}$ and

$$
\frac{\partial N}{\partial \sigma_{k}}=\frac{1}{\sqrt{2 g}} \frac{m_{\chi}^{2}}{m_{\sigma} m_{\mathrm{pl}}^{2}} .
$$

Finally, putting all this together we find

$$
f_{\mathrm{NL}} \simeq \frac{5}{6} \frac{\lambda_{\phi} m_{\mathrm{pl}}^{2}}{m_{\chi}^{2}} .
$$

It must be emphasized this is an upper limit; smaller $f_{\mathrm{NL}}$ are easily achieved by choosing parameters that do not minimize $\left(\partial N / \partial \sigma_{k}\right)^{-2}$. Note also that in this implementation of the model the only constraint on $\lambda_{\phi}$ is that $\lambda_{\phi} \delta \phi^{2}=\lambda_{\phi}\left(H_{k} / \sqrt{2}\right)^{2} \ll m_{\chi}^{2}$. This constraint assures that $\chi$ always rolls away from the origin before $\sigma$ reaches zero and gives

$$
\lambda_{\phi}\left(\frac{1}{12 g} \frac{m_{\chi}^{2}}{m_{\mathrm{pl}}^{2}}\right) \ll 1
$$

Referring to the second constraint of Eqs. (15), we see the term in parenthesis is already constrained to be much less than unity. Therefore this mechanism permits a nonGaussian component to the density perturbations all the way through the observational limit of $f_{\mathrm{NL}} \lesssim 135$ [24].

For the above calculation to be appropriate requires that the $\zeta_{\mathrm{g}}$ of Eq. (29) actually be the dominant contribution to 
the curvature perturbation. For this to be the case its power spectrum must have a magnitude $\mathcal{P}_{\zeta, \mathrm{g}} \approx\left(5 \times 10^{-5}\right)^{2}$ in order to match observation [1]. From Eq. (6) and Eq. (32) we have

$$
\mathcal{P}_{\zeta, \mathrm{g}}=\left(\frac{\partial N}{\partial \sigma_{k}}\right)^{2} \frac{H_{k}^{2}}{4 \pi^{2}}=\frac{1}{8 \pi^{2} \epsilon_{k}} \frac{H_{k}^{2}}{m_{\mathrm{pl}}^{2}},
$$

where we have used that in this scenario the first slow-roll parameter as the mode $k$ exits the horizon is

$$
\epsilon_{k}=\frac{1}{2 m_{\mathrm{pl}}^{2}}\left(\frac{\partial N}{\partial \sigma_{k}}\right)^{-2}=\frac{g m_{\sigma}^{2} m_{\mathrm{pl}}^{2}}{m_{\chi}^{4}} .
$$

We have written the power spectrum in this way in order to employ the observational constraints on the spectral tilt and on the level of gravity waves.

Since the primary, Gaussian density perturbations are now sourced during inflation, the spectral tilt is $n-1=$ $2 \eta-6 \epsilon_{k} \approx 2 \epsilon_{k} \lesssim 0.04$ [1], where we have used that the second slow-roll parameter in the scenario we are considering is given by

$$
\eta \equiv \frac{m_{\mathrm{pl}}^{2}}{V} \frac{\partial^{2} V}{\partial \sigma^{2}} \approx \frac{4 g m_{\sigma}^{2} m_{\mathrm{pl}}^{2}}{m_{\chi}^{4}} \approx 4 \epsilon_{k} .
$$

Meanwhile, it is observed that $H_{k}^{2} / m_{\mathrm{pl}}^{2} \lesssim 10^{-8}$ [20]. In this model we can decrease the spectral tilt independently of $H_{k}^{2} / m_{\mathrm{pl}}^{2}$ by simply decreasing $m_{\sigma}$. Therefore we simply require to set $H_{k}^{2} / m_{\mathrm{pl}}^{2} \lesssim 10^{-9}$ to match observation. Here

$$
\frac{H_{k}^{2}}{m_{\mathrm{pl}}^{2}}=\frac{1}{6 g} \frac{m_{\chi}^{4}}{m_{\mathrm{pl}}^{4}}=\frac{5}{3} \lambda_{\phi} f_{\mathrm{NL}}^{-1}\left(\frac{1}{12 g} \frac{m_{\chi}^{2}}{m_{\mathrm{pl}}^{2}}\right)
$$

where we have expressed the result in terms of $f_{\mathrm{NL}}$ to clarify which are the remaining free parameters. According to the second constraint in Eq. (15), the term in parentheses is already constrained to be much less than unity. In fact, it can be set as small as necessary to satisfy observation. Moreover, since the three terms in Eq. (38) depend upon three independent parameters $\left(\lambda_{\phi}, g\right.$, and $m_{\chi}$ ), we have considerable freedom in exactly how we satisfy the observational bound. Thus we conclude that this model allows for significant non-Gaussianities for a range of model parameters.

\section{DISCUSSION AND CONCLUSIONS}

In this work we continue the analysis of the model proposed in [10] to generate density perturbations at the end of inflation. We confirm that these density perturbations can easily dominate over those produced via the standard inflationary paradigm, and explore the sensitivity of this result to the tuning of model parameters. In addition, we provide a straightforward variation of this model which allows for even greater amplification of the density perturbations.

It is worthwhile to consider how general is this analysis. According to Eq. (7) the production of density perturbations at the end of inflation is most effective when the slowroll parameter near the end of inflation is much less than that when cosmological scales of interest exit the particle horizon. Since inflation can only end with the slow-roll parameter rising to unity, this suggests the mechanism is most effective only when the inflationary potential contains large derivatives near the point where it dips toward its minimum. These large derivatives are most naturally accomplished by inserting a second field direction for the vacuum energy to fall to zero and initiate a reheating phase. This is precisely the scenario implemented in hybrid inflation and generalized in the models considered here.

We also study a variation in the implementation of the model proposed in [10] that results in modified inflationary dynamics. We show that this case results in a spectrum of density perturbations with significant non-Gaussianities for a range of model parameters. In particular it is shown that these non-Gaussianities are capable of saturating the current observational bound.

\section{ACKNOWLEDGMENTS}

M. S. thanks Michael Graesser and Mark Wise for helpful discussions and thanks Michael Graesser for useful comments upon his review of the manuscript. M.S. also thanks David Lyth for correcting an assumption made in an earlier report of this work and for other helpful comments. This work was supported by the U.S. Department of Energy under Contract No. DE-FG03-92ER40701.
[1] C. L. Bennett et al., Astrophys. J. 464, L1 (1996); C. L. Bennett et al., Astrophys. J. Suppl. Ser. 148, 1 (2003); D. N. Spergel et al. (WMAP Collaboration), Astrophys. J. Suppl. Ser. 148, 175 (2003).

[2] A. H. Guth, Phys. Rev. D 23, 347 (1981); A. D. Linde, Phys. Lett. 108B, 389 (1982).

[3] For reviews of standard inflation, see for example S.
Dodelson, Modern Cosmology, (Academic Press, San Diego, 2003); A. D. Linde, hep-th/0503203; D. H. Lyth and A. Riotto, Phys. Rep. 314, 1 (1999).

[4] A. H. Guth and S. Y. Pi, Phys. Rev. Lett. 49, 1110 (1982); S. W. Hawking, Phys. Lett. 115B, 295 (1982); A. A. Starobinsky, Phys. Lett. 117B, 175 (1982); J. M. Bardeen, P. J. Steinhardt, and M. S. Turner, Phys. Rev. D 
28, 679 (1983).

[5] D. H. Lyth and D. Wands, Phys. Lett. B 524, 5 (2002); T. Moroi and T. Takahashi, Phys. Lett. B 522, 215 (2001); 539, 303(E) (2002); K. Enqvist and M. S. Sloth, Nucl. Phys. B626, 395 (2002).

[6] S. Mollerach, Phys. Rev. D 42, 313 (1990); A. D. Linde and V. Mukhanov, Phys. Rev. D 56, R535 (1997).

[7] G. Dvali, A. Gruzinov, and M. Zaldarriaga, Phys. Rev. D 69, 023505 (2004); L. Kofman, astro-ph/0303614.

[8] G. Dvali, A. Gruzinov, and M. Zaldarriaga, Phys. Rev. D 69, 083505 (2004); L. Ackerman, C. W. Bauer, M.L. Graesser, and M. B. Wise, Phys. Lett. B 611, 53 (2005); C. W. Bauer, M. L. Graesser, and M. P. Salem, Phys. Rev. D 72, 023512 (2005); R. Allahverdi, Phys. Rev. D 70, 043507 (2004); T. Matsuda, Phys. Rev. D 72, 123508 (2005).

[9] B. A. Bassett, S. Tsujikawa, and D. Wands, astro-ph/ 0507632.

[10] D. H. Lyth, astro-ph/0510443.

[11] F. Bernardeau, L. Kofman, and J. P. Uzan, Phys. Rev. D 70, 083004 (2004).

[12] J. M. Bardeen, Phys. Rev. D 22, 1882 (1980); J. M. Bardeen, P. J. Steinhardt, and M.S. Turner, Phys. Rev. D 28, 679 (1983).

[13] V.F. Mukhanov, H. A. Feldman, and R. H. Brandenberger, Phys. Rep. 215, 203 (1992); D. Langlois, hep-th/0405053.
[14] M. Sasaki and E. D. Stewart, Prog. Theor. Phys. 95, 71 (1996); A. A. Starobinsky, Phys. Lett. 117B, 175 (1982); A. A. Starobinsky, JETP Lett. 42, 152 (1985) [Pis'ma Zh. Eksp. Teor. Fiz. 42, 124 (1985)]; D. H. Lyth, K. A. Malik, and M. Sasaki, J. Cosmol. Astropart. Phys. 05 (2005) 004.

[15] D. H. Lyth and Y. Rodriguez, Phys. Rev. Lett. 95, 121302 (2005); H. C. Lee, M. Sasaki, E. D. Stewart, T. Tanaka, and S. Yokoyama, J. Cosmol. Astropart. Phys. 10 (2005) 004.

[16] For a pedigogical review of such calculations see for example A. Riotto, hep-ph/0210162.

[17] A. D. Linde, Phys. Lett. B 249, 18 (1990); A. D. Linde, Phys. Rev. D 49, 748 (1994).

[18] A. D. Linde, Phys. Lett. 129B, 177 (1983).

[19] T. S. Bunch and P. C. W. Davies, Proc. R. Soc. A 360, 117 (1978); A. D. Linde, Phys. Lett. 116B, 335 (1982); A. A. Starobinsky and J. Yokoyama, Phys. Rev. D 50, 6357 (1994).

[20] H. V. Peiris et al., Astrophys. J. Suppl. Ser. 148, 213 (2003).

[21] K. Dimopoulos, D. H. Lyth, A. Notari, and A. Riotto, J. High Energy Phys. 07 (2003) 053.

[22] E. Komatsu and D. N. Spergel, Phys. Rev. D 63, 063002 (2001).

[23] J. Maldacena, J. High Energy Phys. 05 (2003) 013.

[24] E. Komatsu et al., Astrophys. J. Suppl. Ser. 148, 119 (2003). 